Supporting Information

\title{
Three-Dimensional Bacterial Motions near a Surface Investigated by Digital Holographic Microscopy: Effect of Surface Stiffness
}

Qingmei Peng, Xin Zhou, Zhi Wang, Qingyi Xie, Chunfeng Ma, Guangzhao Zhang, and Xiangjun Gong*

Faculty of Materials Science and Engineering, South China University of Technology, Guangzhou 510640, P. R. China
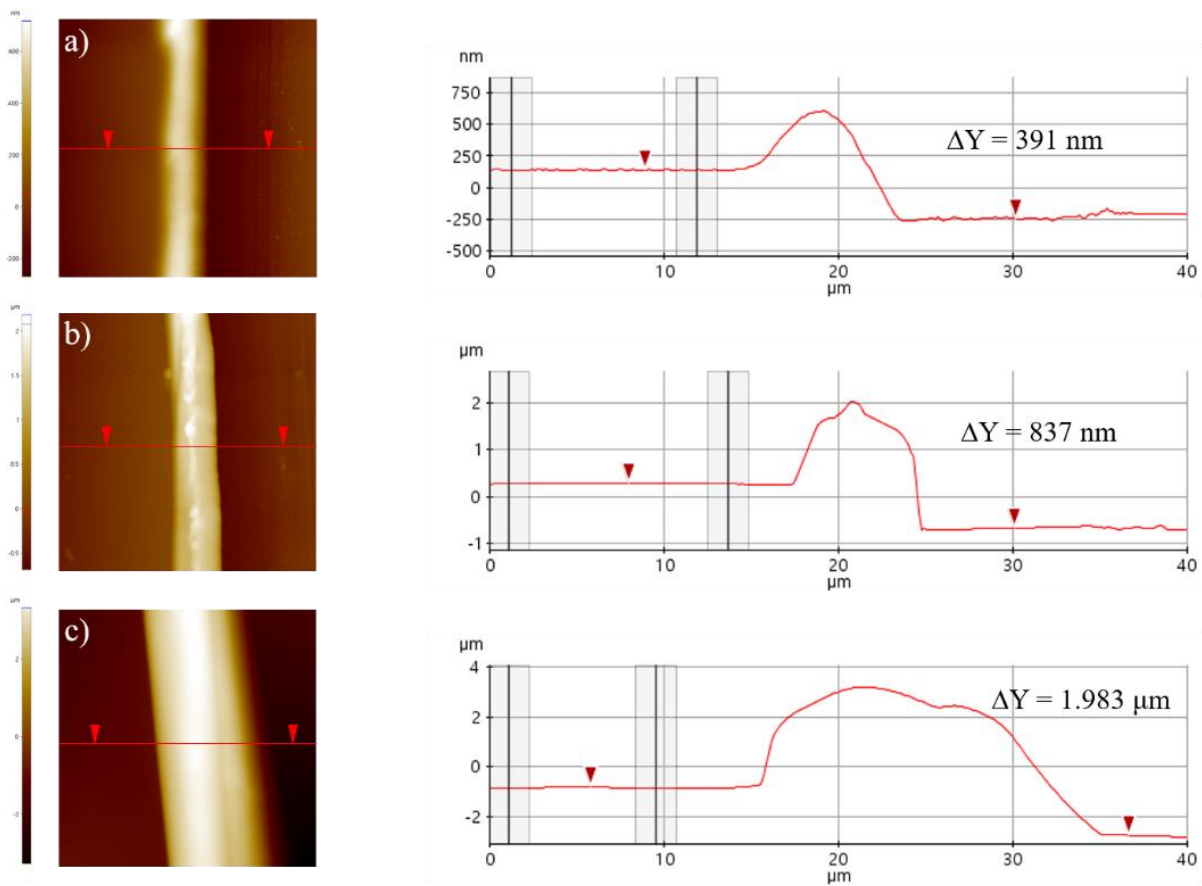

Figure S1. Thickness of the PDMS coatings measured by AFM. The PDMS layers are 0.4, 0.8, and $2.0 \mu \mathrm{m}$, respectively. 

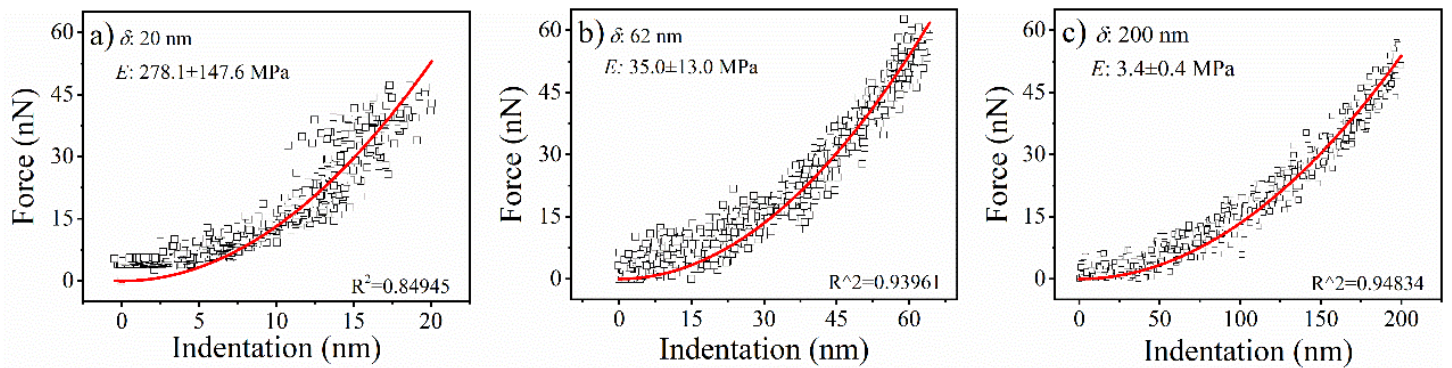

Figure S2. Force-indentation curves for the PDMS surfaces with different stiffness. The scatter plots represent the linear elasticity region of the measured force-indentation curves, and the red curves are the curves fit with the Hertzian model. Young's modulus $(E)$ and corresponding indentation depth $(\delta)$ were obtained from the fitting.
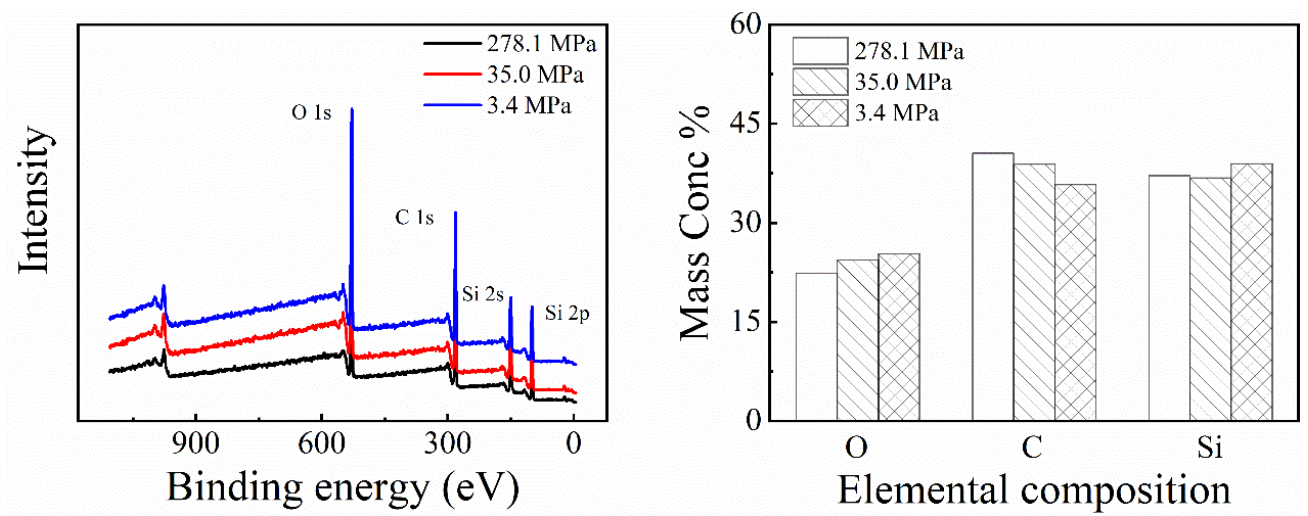

Figure S3. XPS scan spectra and elemental mass concentration analysis of the PDMS surfaces with different stiffness. The surface stiffness does not make difference in the surface composition.

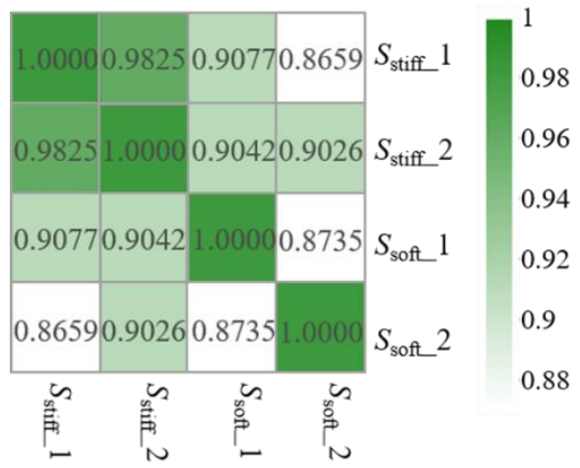

Figure S4. Correlation heat map of the two repetitive experiments within the group. As the color 
of the heat map darkens, the Pearson correlation closer to 1, indicating a better biological repeatability.
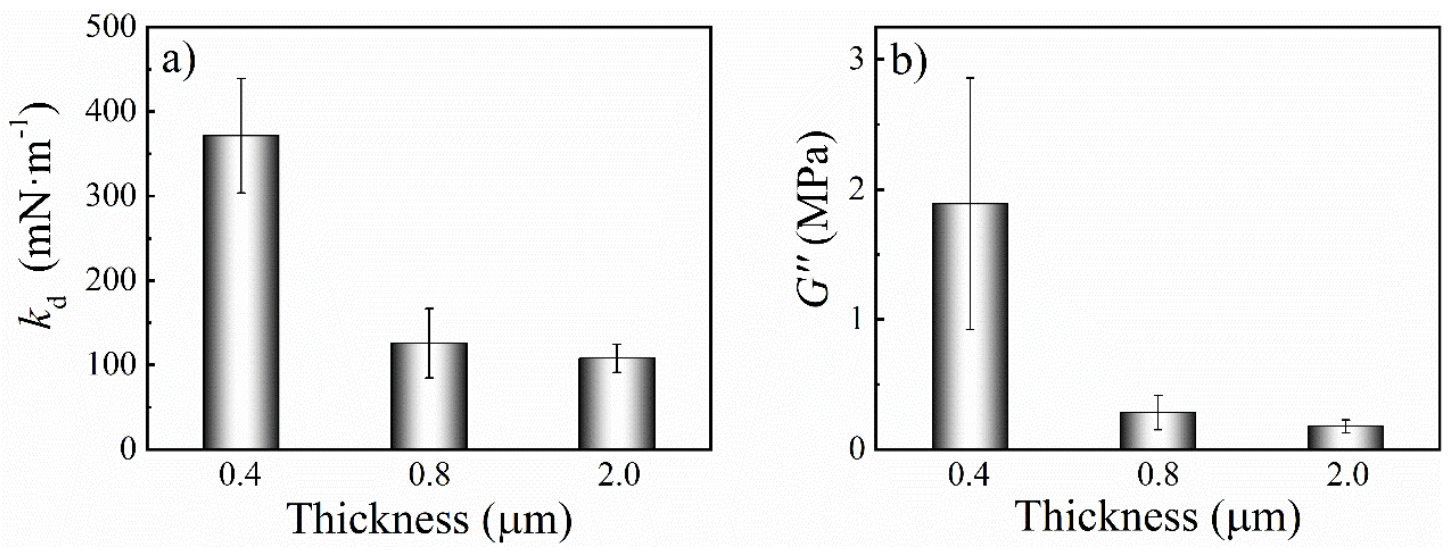

Figure S5. Dynamic stiffness $\left(k_{\mathrm{d}}\right)$ and loss modulus $\left(G^{\prime \prime}\right)$ of the PDMS surfaces with different thickness were measured by magnetic force modulation atomic force microscopy (MF-AFM) with frequency $f=5 \mathrm{~Hz}$.

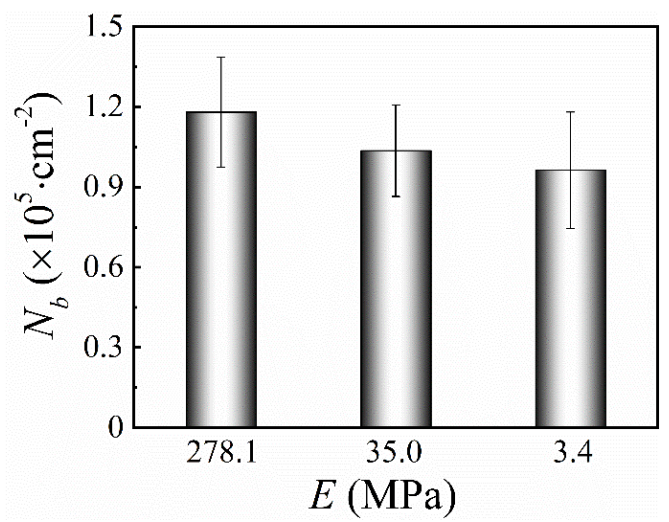

Figure S6. The number $\left(N_{\mathrm{b}}\right)$ of $E$. coli HCB1414 adhered onto the PDMS surfaces with different stiffness. 


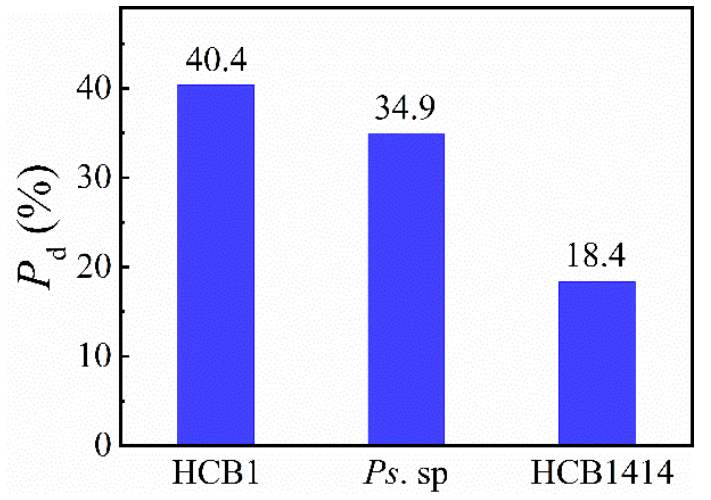

Figure S7. Decreased percentage $\left(P_{\mathrm{d}}\right)$ of the adhered number $\left(N_{\mathrm{b}}\right) . P_{\mathrm{d}}$ is calculated by $\left(N_{\mathrm{b}}\right.$ (stiff) $N_{\mathrm{b} \text { (soft) }} / N_{\mathrm{b} \text { (stiff). }}$ The subscripts (stiff) and (soft) refer to the bacteria upon surfaces with $E=278.1$ and 3.4 MPa, respectively.

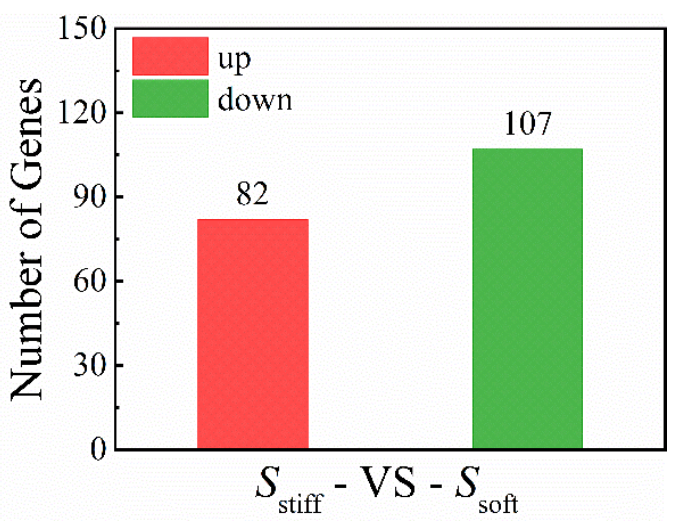

Figure S8. Statistics of differential expression genes of $E$. coli HCB1 upon the softer surface $(E=$ 3.4 MPa) compared to the stiffer surface (278.1 MPa).

Signal Diguanylate cyclase

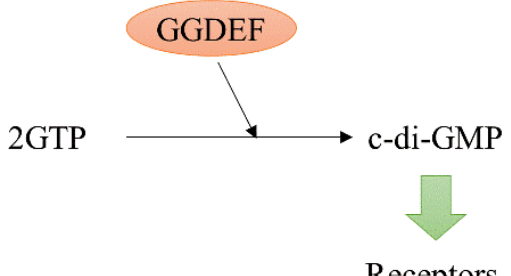

Phosphodiesterase Signal

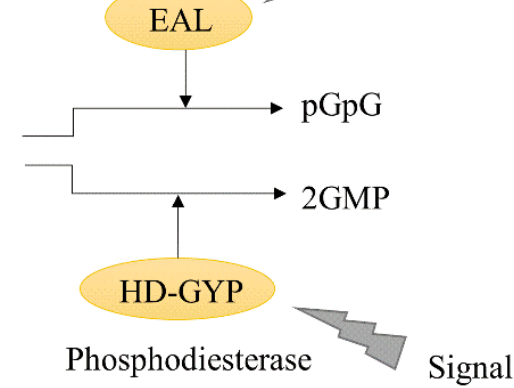


Figure S9. A model of c-di-GMP molecular regulation module. c-di-GMP is produced by DGC proteins containing the GGDEF domain and degraded by PDE proteins bearing the EAL or HD GYP domains.

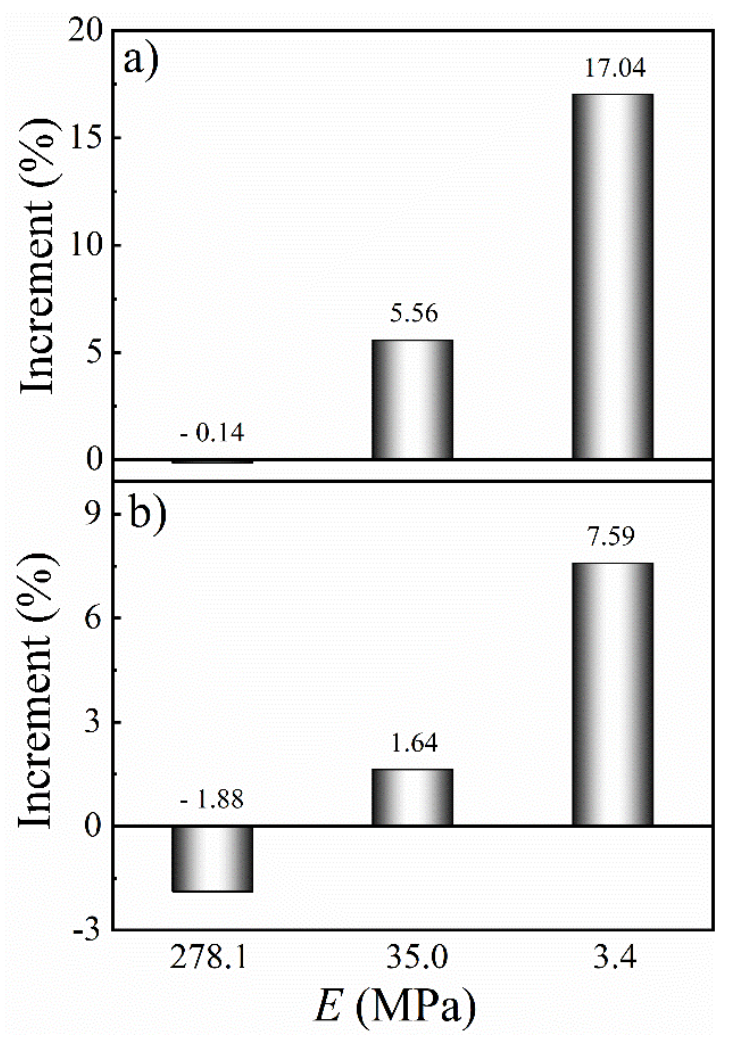

Figure S10. Comparison of the increment of average $V_{3 \mathrm{D}}$ of (a) E. coli HCB1 and (b) Pseudomonas sp. from $z=10 \mu \mathrm{m}\left(V_{10 \mu \mathrm{m}}\right)$ to $1 \mu \mathrm{m}\left(V_{1 \mu \mathrm{m}}\right)$, which is calculated as: $\left(V_{1 \mu \mathrm{m}}-V_{10}\right.$ $\mu \mathrm{m}) / V_{10 \mu \mathrm{m}} \times 100 \%$.

\section{Supplementary Methods}

Viscoelasticity Measurements. Viscoelasticity of PDMS surfaces with different thickness was measured with magnetic force modulation atomic force microscopy (MF-AFM). A magnetic cantilever was used to penetrate into samples and oscillate around an initial indentation $(\delta)$ at a certain frequency $(f)$ in the surface normal 
direction. In this way, the amplitude and shif of the cantilever deflection could be used to extract the viscoelasity of the samples. The details about the preparing of the magnetic probe and procedure of measurement were described elsewhere. ${ }^{1,2}$ The spring constant $\left(k_{\mathrm{c}}\right)$ of this probe was calibrated by the thermal tune method, and the viscoelasticity measurements of the surfaces were performed at $25{ }^{\circ} \mathrm{C}$ in air. The measurements were taken three times at different positions. The dynamic stiffness $\left(k_{\mathrm{d}}\right)$ were calculated by the following equation:

$$
k_{d}=k_{c}\left(\frac{A_{0}}{A_{1}}-1\right)
$$

In Eq. S1, $A_{0}$ and $A_{1}$ are free amplitude (in air) and amplitude near the surface. The storage modulus $\left(G^{\prime}\right)$ and loss modulus $\left(G^{\prime \prime}\right)$ can be obtained from dynamic stiffness $\left(k_{\mathrm{d}}\right)$ and phase shift $(\varphi)$ between samples and air with the known indentation $(\delta)$ and Poisson's ratio $(v)$ :

$$
\begin{aligned}
& G^{\prime}=k_{d} \frac{1-v}{2 \sqrt{2} \tan \alpha \delta} \cos \varphi \\
& G^{\prime \prime}=k_{d} \frac{1-v}{2 \sqrt{2} \tan \alpha \delta} \sin \varphi
\end{aligned}
$$

Note that Eqs. S2 and S3 are derived from the Hertzian contact model with Eq. 1. The viscoelasticity can be described by the dynamic stiffness $\left(k_{\mathrm{d}}\right)$. Especially, the viscosity of samples can be further described by the loss modulus $\left(G^{\prime \prime}\right)$ of the surface at a certain frequency.

\section{REFERENCES}

(1) Gan, T.; Gong, X.; Schonherr, H.; Zhang, G. Microrheology of growing Escherichia coli biofilms investigated by using magnetic force modulation atomic 
force microscopy. Biointerphases 2016, 11 (4), 041005.

(2) Wang, Z.; Gong, X.; Xie, J.; Xu, Z.; Liu, G.; Zhang, G. Investigation of Formation of Bacterial Biofilm upon Dead Siblings. Langmuir 2019, 35 (23), 7405-7413. 